RYSZARD ZiELIŃSKI (Warszawa)

\title{
ESTIMATING QUANTILES WITH LINEX LOSS FUNCTION. APPLICATIONS TO VaR ESTIMATION
}

Abstract. Sometimes, e.g. in the context of estimating VaR (Value at Risk), underestimating a quantile is less desirable than overestimating it, which suggests measuring the error of estimation by an asymmetric loss function. As a loss function when estimating a parameter $\theta$ by an estimator $T$ we take the well known Linex function $\exp \{\alpha(T-\theta)\}-\alpha(T-\theta)-1$. To estimate the quantile of order $q \in(0,1)$ of a normal distribution $N(\mu, \sigma)$, we construct an optimal estimator in the class of all estimators of the form $\bar{x}+k \sigma,-\infty<k<\infty$, if $\sigma$ is known, or of the form $\bar{x}+\lambda s$, if both parameters $\mu$ and $\sigma$ are unknown; here $\bar{x}$ and $s$ are the standard estimators of $\mu$ and $\sigma$, respectively. To estimate a quantile of an unknown distribution $F$ from the family $\mathcal{F}$ of all continuous and strictly increasing distribution functions we construct an optimal estimator in the class $\mathcal{T}$ of all estimators which are equivariant with respect to monotone transformations of data.

1. The problem. In some applications underestimating a quantile is less desirable than overestimating it. That is the case, though not commonly recognized, in the problem of estimating VaR (Value at Risk) (Khindanova et al. 2000, Yi-Ping Chang et al. 2003). Consequences of fixing VaR too low are much more serious that consequences of fixing it at a too high level. Formally the problem of estimating VaR may be stated as the problem of constructing an estimator which minimizes the risk of estimation under a Linex loss function, which for an estimator $T$ and and an estimand $\theta$ takes on the form $\exp \{\alpha(T-\theta)\}-\alpha(T-\theta)-1$ (Fig. 1).

2000 Mathematics Subject Classification: 62F10, 62 G05.

Key words and phrases: quantile estimation, Linex loss, VaR (Value at Risk), normal distribution, nonparametric model. 


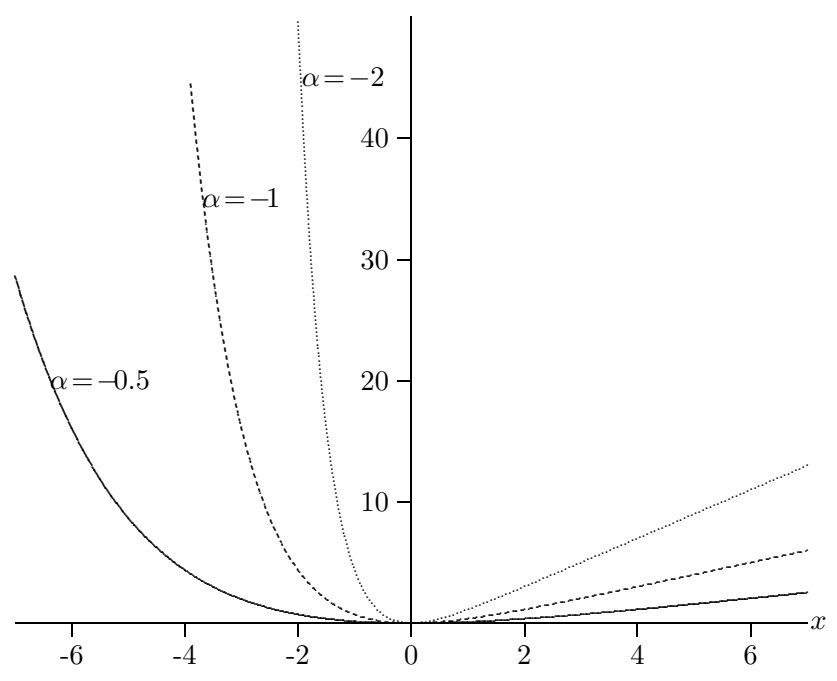

Fig. 1. $\exp \{\alpha x\}-\alpha x-1$

In what follows we construct an optimal estimator in the normal model and in a nonparametric model on the basis of a random sample $x_{1}, \ldots, x_{n}$ (i.i.d. observations) with a fixed sample size $n$ (non-asymptotic solution).

2. Estimating quantiles of a normal distribution. Given a sample $x_{1}, \ldots, x_{n}$ from a normal distribution $N(\mu, \sigma)$, the problem is to estimate the $q$ th quantile $x_{q}(\mu, \sigma)=\mu+z_{q} \sigma$, where $z_{q}=\Phi^{-1}(q)$ and $\Phi$ is the distribution function of $N(0,1)$.

We consider the class of all estimators of the form $\bar{x}+k \sigma,-\infty<k<\infty$, if $\sigma$ is known, or of the form $\bar{x}+\lambda s$ if both parameters $\mu$ and $\sigma$ are unknown. Here

$$
\bar{x}=\frac{1}{n} \sum_{j=1}^{n} x_{j} \quad \text { and } \quad s^{2}=\frac{1}{n} \sum_{j=1}^{n}\left(x_{j}-\bar{x}\right)^{2}
$$

are the standard estimators of $\mu$ and $\sigma$ with probability distribution functions

$$
f(\bar{x})=\frac{\sqrt{n}}{\sigma \sqrt{2 \pi}} \exp \left\{-\frac{n}{2}\left(\frac{\bar{x}-\mu}{\sigma}\right)^{2}\right\}
$$

and

$$
g(s)=\frac{2}{\sigma \Gamma\left(\frac{n-1}{2}\right)}\left(\frac{n}{2}\right)^{(n-1) / 2}\left(\frac{s}{\sigma}\right)^{n-2} \exp \left\{-\frac{n}{2}\left(\frac{s}{\sigma}\right)^{2}\right\},
$$

respectively.

3. Optimal estimator if $\sigma$ is known. As a measure of discrepancy between the $q$ th quantile $x_{q}(\mu, \sigma)$ to be estimated and the estimator $\bar{x}+k \sigma$ 
we take the Linex loss function in the form (Fig. 1)

$L_{0}(\bar{x}, k, q, n, \alpha)=\exp \left\{\alpha \frac{(\bar{x}+k \sigma)-x_{q}(\mu, \sigma)}{\sigma}\right\}-\alpha \frac{(\bar{x}+k \sigma)-x_{q}(\mu, \sigma)}{\sigma}-1$.

Theorem 1. Assuming the loss function $L_{0}(\bar{x}, k, q, n, \alpha)$, the optimal estimator of the qth quantile $x_{q}(\mu, \sigma)$, if $\sigma$ is known, is of the form

$$
\bar{x}+\left(z_{q}-\alpha / 2 n\right) \sigma .
$$

Proof. The risk function of the estimator $\bar{x}+k \sigma$ under the Linex loss $L_{0}(\bar{x}, k, q, n, \alpha)$ is given by the formula

$$
\begin{aligned}
R_{0}(k, q, n, \alpha) & =\int_{-\infty}^{\infty} L_{0}(\bar{x}, k, q, n, \alpha) f(\bar{x}) d \bar{x} \\
& =\exp \left\{\alpha\left(k-z_{q}\right)+\frac{\alpha^{2}}{2 n}\right\}-\alpha\left(k-z_{q}\right)-1 .
\end{aligned}
$$

Minimization of the risk with respect to $k$ gives us the optimal estimator $\bar{x}+k \sigma$ with $k=k(q, n, \alpha)=z_{q}-\alpha / 2 n$.

4. Optimal estimator if both $\mu$ and $\sigma$ are unknown. As a measure of discrepancy between the $q$ th quantile $x_{q}(\mu, \sigma)$ to be estimated and the estimator $\bar{x}+k s$ we take the Linex loss function in the form $L(\bar{x}, s, q, \lambda, n, \alpha)=\exp \left\{\alpha \frac{(\bar{x}+\lambda s)-x_{q}(\mu, \sigma)}{\sigma}\right\}-\alpha \frac{(\bar{x}+\lambda s)-x_{q}(\mu, \sigma)}{\sigma}-1$.

Theorem 2. Assuming the loss function $L(\bar{x}, s, \lambda, q, n, \alpha)$, the optimal estimator of the qth quantile $x_{q}(\mu, \sigma)$, if both $\mu$ and $\sigma$ are unknown, is of the form

$$
\bar{x}+\lambda s,
$$

where $\lambda=\lambda(q, n, \alpha)$ is the unique solution of the equation

$$
\int_{0}^{\infty} t^{n-1} \exp \left\{\alpha \lambda t-\frac{n}{2} t^{2}\right\} d t=\frac{1}{2}\left(\frac{2}{n}\right)^{n / 2} \Gamma\left(\frac{n}{2}\right) \exp \left\{-\alpha\left(\frac{\alpha}{2 n}-z_{q}\right)\right\} .
$$

Comment. The left hand side of the above equation is well known as the Parabolic Cylinder Function or Weber function, which is related to confluent hypergeometric functions or Whittaker functions (e.g. Abramowitz and Stegun 1972 or Gradshteyn and Ryzhik 2000). This enables us to use standard tables or computer packages for calculating $\lambda$.

Proof. The risk function of the estimator $\bar{x}+\lambda s$ under the Linex loss $L(\bar{x}, s, q, \lambda, n, \alpha)$ is given by the formula

$$
R(\lambda, q, n, \alpha)=\int_{-\infty}^{\infty} d \bar{x} \int_{0}^{\infty} d s L(\bar{x}, s, q, \lambda, n, \alpha) f(\bar{x}) g(s) .
$$


Now,

$$
\begin{aligned}
& \int_{-\infty}^{\infty} \exp \left\{\alpha \frac{\bar{x}-\mu}{\sigma}\right\} f(\bar{x}) d \bar{x}=\frac{\sqrt{n}}{\sqrt{2 \pi}} \int_{-\infty}^{\infty} \exp \left\{\alpha t-\frac{n}{2} t^{2}\right\} d t=\exp \left\{\frac{\alpha^{2}}{2 n}\right\} \\
& \int_{0}^{\infty} \exp \left\{\alpha \frac{\lambda s-z_{q} \sigma}{\sigma}\right\} g(s) d s=\exp \left\{-\alpha z_{q}\right\} \int_{0}^{\infty} \exp \left\{\alpha \lambda \frac{s}{\sigma}\right\} g(s) d s \\
& =\frac{2}{\sigma \Gamma\left(\frac{n-1}{2}\right)}\left(\frac{n}{2}\right)^{(n-1) / 2} \exp \left\{-\alpha z_{q}\right\} \int_{0}^{\infty} t^{n-2} \exp \left\{\alpha \lambda t-\frac{n}{2} t^{2}\right\} d t \\
& \int_{-\infty}^{\infty} \alpha \frac{\bar{x}-\mu}{\sigma} f(\bar{x}) d \bar{x}=0 \\
& \int_{0}^{\infty} \alpha\left(\lambda \frac{s}{\sigma}-z_{q}\right) g(s) d s=\alpha\left(\lambda \sqrt{\frac{2}{n}} \frac{\Gamma\left(\frac{n}{2}\right)}{\Gamma\left(\frac{n-1}{2}\right)}-z_{q}\right)
\end{aligned}
$$

and hence

$$
\begin{aligned}
R(\lambda, q, n, \alpha) & =\frac{2}{\sigma \Gamma\left(\frac{n-1}{2}\right)}\left(\frac{n}{2}\right)^{(n-1) / 2} \exp \left\{\alpha\left(\frac{\alpha}{2 n}-z_{q}\right)\right\} \int_{0}^{\infty} t^{n-2} \exp \left\{\alpha \lambda t-\frac{n}{2} t^{2}\right\} d t \\
& -\alpha\left(\sqrt{\frac{2}{n}} \frac{\Gamma\left(\frac{n}{2}\right)}{\Gamma\left(\frac{n-1}{2}\right)} \lambda-z_{q}\right)-1 .
\end{aligned}
$$

The first summand of the risk $R(\lambda, q, n, \alpha)$ is strictly decreasing in $\lambda$ and the second summand is strictly increasing, so there exists exactly one $\lambda$ which minimizes the risk, and that is the solution of the equation $\partial R(\lambda, q, n, \alpha) / \partial \lambda$ $=0$.

Some numerical values of optimal $k$ for the case of known $\sigma$ and optimal $\lambda$ for the case of both parameters of the parent distribution $N(0,1)$ unknown are presented in Table 1.

It is obvious that $k(n, q, \alpha) \rightarrow z_{q}$ as $n \rightarrow \infty$. Though numerically easily confirmed, no analytic proof of the convergence $\lambda(n, q, \alpha) \rightarrow z_{q}$ as $n \rightarrow \infty$ is known to the author.

\section{Estimating quantiles of an unknown distribution $F$ from} a large nonparametric family $\mathcal{F}$. Let $\mathcal{F}$ be the family of all continuous and strictly increasing (on their supports) distribution functions and let $x_{q}(F)=F^{-1}(q)$ be the (unique) $q$ th quantile (quantile of order $q$ ) of the distribution $F \in \mathcal{F}$. Let $X_{1: n}, X_{2: n}, \ldots, X_{n: n}\left(\right.$ with $\left.X_{1: n} \leq X_{2: n} \leq \cdots \leq X_{n: n}\right)$ be an ordered sample from an unknown distribution $F \in \mathcal{F}$. The sample size $n$ is assumed to be fixed. The problem is to estimate $x_{q}(F)$. 
Table 1. Optimal values of $k$ (first row) and $\lambda$ (second row)

\begin{tabular}{|l|l|l|l|l|l|}
\hline \multirow{3}{*}{$q$} & \multirow{2}{*}{$n$} & \multicolumn{4}{|c|}{$\alpha$} \\
\cline { 3 - 6 } & & -0.5 & -1 & \multicolumn{1}{|c|}{-2} & \multicolumn{1}{|c|}{-5} \\
\hline \multirow{3}{*}{0.5} & 10 & 0.025 & 0.05 & 0.1 & 0.25 \\
& & 0.02564 & 0.05133 & 0.10306 & 0.26487 \\
\cline { 2 - 6 } & 20 & 0.0125 & 0.025 & 0.05 & 0.125 \\
& & 0.01266 & 0.02532 & 0.05069 & 0.12758 \\
\cline { 2 - 6 } & 50 & 0.005 & 0.01 & 0.02 & 0.05 \\
& & 0.00503 & 0.01005 & 0.02010 & 0.05031 \\
\cline { 2 - 6 } & 100 & 0.0025 & 0.005 & 0.01 & 0.025 \\
& & 0.00251 & 0.00501 & 0.01003 & 0.02507 \\
\hline \multirow{3}{*}{0.9} & 10 & 1.30655 & 1.33155 & 1.38155 & 1.53155 \\
& & 1.36246 & 1.41387 & 1.52668 & 1.97887 \\
\cline { 2 - 6 } & 20 & 1.29405 & 1.30655 & 1.33155 & 1.40655 \\
& & 1.32117 & 1.34533 & 1.39582 & 1.56758 \\
\cline { 2 - 6 } & 50 & 1.28655 & 1.29155 & 1.30155 & 1.33155 \\
& & 1.29720 & 1.30652 & 1.32549 & 1.38507 \\
\cline { 2 - 6 } & 100 & 1.28405 & 1.28655 & 1.29155 & 1.30655 \\
& & 1.28934 & 1.29395 & 1.30324 & 1.33174 \\
\hline
\end{tabular}

As a class $\mathcal{T}$ of estimators to be considered we take the class of all estimators which are equivariant with respect to monotonic transformations of data, and we measure the error of estimation of $x_{q}(F)$ by an estimator $T \in \mathcal{T}$ by means of differences $F(T)-q$; the rationale for such a choice can be found, for example, in Zieliński $(1999,2001,2004)$. The Linex loss function takes on the form $\exp \{\alpha(F(T)-q)\}-\alpha(F(T)-q)-1, \alpha<0$ (Fig. 2).
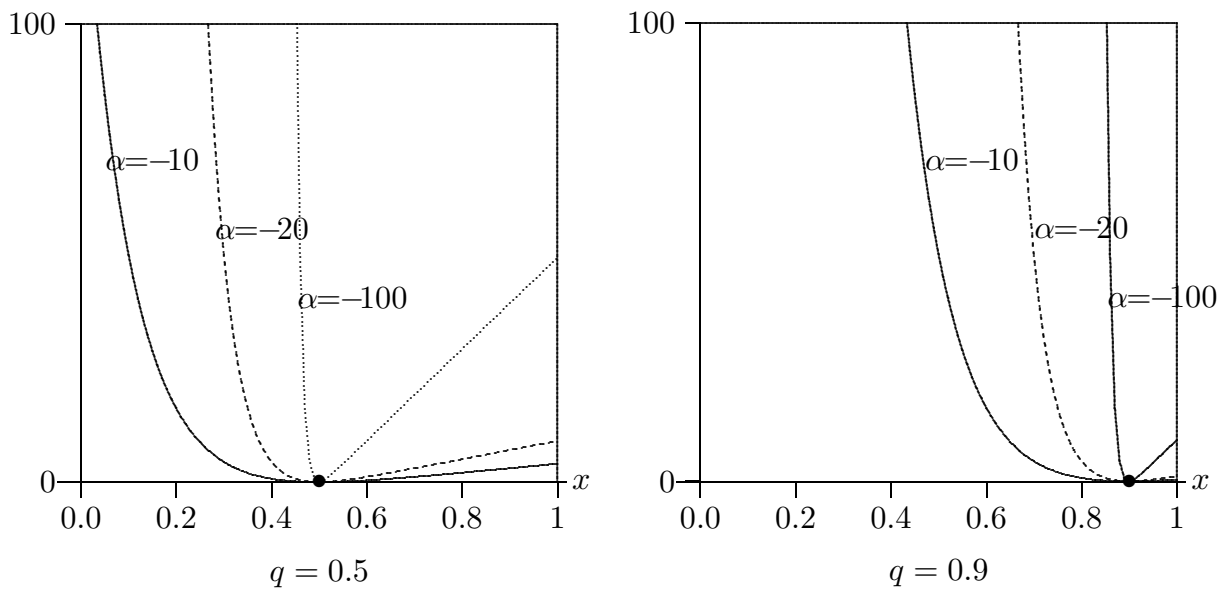

Fig. 2. $\exp \{\alpha(x-q)-\alpha(x-q)-1\}$ 
An estimator $T$ belongs to the class $\mathcal{T}$ iff it is of the form $T=X_{J(\lambda): n,}$, where $J=J(\lambda)$ is a random integer, independent of the sample $X_{1: n}, \ldots$ $\ldots, X_{n: n}$, such that $P\{J=j\}=\lambda_{j}, \sum \lambda_{j}=1, \lambda_{j} \geq 0$ (Uhlmann 1963 for $j$ fixed, Zieliński 2004 for $J$ random). Observe that if the sample comes from a distribution $F \in \mathcal{F}$ then $F(T)=F\left(X_{J(\lambda): n}\right)=U_{J(\lambda): n}$, where $U_{j: n}$ is the $j$ th order statistic from the uniform distribution $U(0,1)$. It follows that the risk of the estimator $T=X_{J(\lambda): n}$ under the Linex loss is given by the sum

where

$$
\sum_{j=1}^{n} \lambda_{j} R(j, n ; q, \alpha)
$$

$$
\begin{aligned}
& R(j, n ; q, \alpha) \\
& \quad=\frac{n !}{(j-1) !(n-j) !} \int_{0}^{1}(\exp \{\alpha(x-q)\}-\alpha(x-q)-1) x^{j-1}(1-x)^{n-j} d x \\
& \quad=e^{-\alpha q}{ }_{1} F_{1}(j, n+1 ; \alpha)-\frac{j}{n+1} \alpha+(\alpha q-1) .
\end{aligned}
$$

Here

$$
{ }_{1} F_{1}(j, n+1 ; \alpha)=\frac{\Gamma(n+1)}{\Gamma(j) \Gamma(n-j+1)} \int_{0}^{1} e^{\alpha t} t^{j-1}(1-t)^{n-j} d t
$$

is the confluent hypergeometric function (e.g. Weisstein 1999, Luke 1975). Using the recurrence relation

$$
{ }_{1} F_{1}(j, n+1 ; \alpha)-{ }_{1} F_{1}(j-1, n+1 ; \alpha)=\frac{\alpha}{n+1}{ }_{1} F_{1}(j, n+2 ; \alpha)
$$

and taking into account that ${ }_{1} F_{1}(j, n+1 ; \alpha)>0$ we conclude that the first term in $R(j, n ; q, \alpha)$ is decreasing in $j$, the term $j /(n+1)$ is obviously increasing and as a result the optimal estimator is of the form $X_{j^{*}: n}$ with $j^{*}$ such that

$$
R\left(j^{*}, n ; q, \alpha\right)=\min _{j} R(j, n ; q, \alpha) .
$$

It follows that for $j \in\{1, \ldots, n\}$ there exists a unique $j^{*}$ such that

$$
R\left(j^{*}, n ; \alpha, q\right)<R(j, n ; \alpha, q), \quad j \neq j^{*},
$$

or

$$
R\left(j^{*}, n ; \alpha, q\right)=R\left(j^{*}+1, n ; q, \alpha\right)<R(j, n ; q, \alpha), \quad j \notin\left\{j^{*}, j^{*}+1\right\} .
$$

The optimal $j^{*}$ can be easily found numerically. Some values of $j^{*}=$ $j^{*}(n, \alpha, q)$ are presented in Table 2 . 
Table 2. Optimal $j^{*}(n, \alpha, q) ; q=0.5$-first row, $q=0.9$ - second row

\begin{tabular}{|c|r|r|r|r|c|}
\hline \multirow{2}{*}{$n$} & \multicolumn{5}{|c|}{$\alpha$} \\
\cline { 2 - 6 } & -1 & -10 & -20 & -50 & -100 \\
\hline \multirow{2}{*}{10} & 6 & 7 & 10 & 10 & 10 \\
& 10 & 10 & 10 & 10 & 10 \\
\hline \multirow{2}{*}{20} & 11 & 12 & 13 & 16 & 20 \\
& 19 & 20 & 20 & 20 & 20 \\
\hline 50 & 26 & 27 & 28 & 32 & 37 \\
& 46 & 47 & 47 & 49 & 50 \\
\hline \multirow{2}{*}{100} & 51 & 52 & 53 & 57 & 63 \\
& 91 & 92 & 92 & 94 & 97 \\
\hline
\end{tabular}

\section{References}

M. Abramowitz and I. A. Stegun (eds.) (1972), Handbook of Mathematical Functions with Formulas, Graphs, and Mathematical Tables, Dover, New York.

I. S. Gradshteyn and I. M. Ryzhik (2000), Tables of Integrals, Series, and Products, 6th ed., Academic Press, San Diego, CA.

I. N. Khindanova and S. T. Rachev (2000), Value at risk: recent advances, in: G. Anastassiou (ed.), Handbook of Analytic-Computational Methods in Applied Mathematics, Chapman \& Hall/CRC, Boca Raton, 801-858.

Y. P. Chang, M. C. Hung and Y. F. Wu (2003), Nonparametric estimation for risk in value-at-risk estimator, Comm. Statist. Simulation Comput. 32, 1041-1064.

Y. L. Luke (1975), Mathematical Functions and Their Approximations, Academic Press.

W. Uhlmann (1963), Ranggrössen als Schätzfunktionen, Metrika 7, 339-352.

E. W. Weisstein (1999), Confluent hypergeometric function of the first kind, MathWorldA Wolfram Web Resource, http://mathworld.wolfram.com/ConfluentHypergeometricFunctionoftheFirstKind.html.

R. Zieliński (1999), Best equivariant nonparametric estimator of a quantile, Statist. Probab. Lett. 45, 79-84.

R. Zieliński (2001), PMC-optimal nonparametric quantile estimator, Statistics 35, 453462.

R. Zieliński (2004), Optimal quantile estimators; Small sample approach, preprint 653, IMPAN, November 2004, www.impan.gov.pl/rziel.

Institute of Mathematics

Polish Academy of Sciences

P.O. Box 21

00-956 Warszawa, Poland

E-mail: R.Zielinski@impan.gov.pl

Received on 11.4.2005 